\title{
1 An efficient LBM-DEM simulation method for suspensions of deformable
}

\section{2 preformed particle gels}

3 Kang Zhou ${ }^{\mathrm{a}, \mathrm{b}}$, Jian Hou ${ }^{\mathrm{a}, \mathrm{b}, *}$, Qicheng Sun ${ }^{\mathrm{c}}$, Lanlei Guo ${ }^{\mathrm{d}}$, Shaoxian Bing ${ }^{\mathrm{d}}$, Qingjun Du ${ }^{\mathrm{b}}$, Chuanjin Yao

$4{ }^{a}$ State Key Laboratory of Heavy Oil Processing, China University of Petroleum, Qingdao, 266580,China

$5 \quad{ }^{b}$ School of Petroleum Engineering, China University of Petroleum, Qingdao, 266580, China

$6{ }^{c}$ State Key Laboratory of Hydroscience \& Engineering, Tsinghua University, Beijing 100084, China

$7{ }^{d}$ Exploration and Development Research Institute, Shengli Oilfield Co. Ltd., Sinopec, Dongying 257000, China

8 Abstract: Because of the capacity of in-depth plugging and flowing diversion, the deformable preformed 9 particle gel (PPG) is used as an effective solution to severe fluid channeling and low sweep efficiency in oil 10 development. However, the transport, plugging and deformation of PPG are a complex issue that involves both 11 general characteristics of particle suspension and special deformation process. In addition, the flow of 12 deformable PPG in porous media cannot be simulated by the classical seepage flow theory based on continuum 13 assumptions. Thus, the paper develops an efficient simulation method for suspensions of deformable PPG, 14 which combines the discrete idea of immersed boundary (IB) for particle deformation, lattice Boltzmann 15 method (LBM) for fluid flow, discrete element method (DEM) for particle contact and immersed moving boundary (IMB) method for the solid-fluid interaction. The improved method is first validated by matching of 17 the transport, plugging and deformation of PPG in porous media between numerical simulations and 18 microscopic visualization experiments. Next, the method is used to study the effect of the particle-throat 19 diameter ratio and elastic modulus on the critical pressure gradient, which is the smallest pressure gradient for a 20 given PPG to pass through a throat of the porous media. The results indicate that there is a good exponential 21 relationship between the critical pressure gradient of the PPG and the particle-throat diameter ratio. The critical 22 pressure gradient is also linearly related to the elastic modulus of PPG. Finally, a multivariate mathematical 23 model is proposed to characterize the quantitative relationships among the critical pressure gradient, 24 particle-throat diameter ratio and elastic modulus. The proposed model is validated by comparisons between simulation results and prediction results by the quantitative model. Thus, the model can be used as the most 26 important parameter for macroscopic simulations of PPG flooding in large oilfield-scale projects.

27 Keywords: deformable particle suspension; preformed particle gel; microscopic flow simulation; lattice 28 Boltzmann method; discrete element method

* Corresponding author. Tel.: 86-546-8395660; Fax.: 86-546-8395660.

E-mail address: houjian@upc.edu.cn (J. Hou). 
Most oilfields in China have small sweep efficiency and low oil recovery because of the strong reservoir heterogeneity and severe fluid channeling. In fact, water flooding only produces approximately $30 \%$ of the original oil in place (OOIP) (Kamal et al., 2015). Even after polymer flooding in high water-cut oilfields, approximately 50\% of OOIP remains in the reservoir (Wang et al., 2009). One of the promising enhanced oil recovery (EOR) methods is to block the fluid channeling paths using the deformable preformed particle gel (PPG), which forces the injected fluid to flow through the unswept region of the reservoir that contains crude oil (Imqam and Bai, 2015). More specifically, most PPG particles first transport in the fluid channeling paths, through which a large fraction of the suspension flows, and they can be captured in the throats with smaller diameters. As a result, the subsequent water is forced to change direction and flow towards the original unswept region. The pressure difference across the plugged throat gradually increases with the increase in flow resistance. When the pressure difference increases to a certain value, which is related to the particle and rock properties, the captured PPG begins to deform and finally passes through the throat. Then, the flow paths are redistributed, which improves the overall sweep efficiency. The deformable PPG has been successfully used in the pilot tests of Shengli Oilfield in China (Cui, 2011). However, a more effective suspension design remains a difficult problem because of the limited understanding of the complex flow mechanisms of PPG suspension, which involves the general characteristics of particle suspension and special deformation process.

Field applications and core flooding experiments of deformable PPG have monitored the fluctuation of the injection pressure (Goudarzi et al., 2015; Yao et al., 2015) and flow variation in parallel-sandpack models (Sang et al., 2014). Microscopic visualization experiments have also observed the deformation process of deformable particles in the pore-throat (Yao et al., 2014). However, being limited to the instruments and research scale of experiments, the accurate critical conditions are difficult to obtain, where the PPG can deform and pass through a given throat. Therefore, simulation studies on the flow of PPG suspension are essential. The earlier simulation studies on PPG flooding are generally conducted at macroscopic scale based on the classical seepage theory or size-exclusion method (Bedrikovetsky, 2008; You et al., 2013; Wang et al., 2013; Liu et al., 2017). In these studies, the suspension is treated as a homogeneous solution characterized by the concentration. They cannot directly simulate the flow and deformation of each PPG particle in porous media. To address this problem, the paper develops an efficient microscopic simulation method for deformable PPG based on accurate force analysis. This method involves simulations of the fluid flow, particle contact, solid-fluid interaction and particle 
deformation.

The fluid flow is generally simulated by computational fluid dynamics (CFD) methods (Raynal et al., 2015) and particle methods (Liu and Liu, 2010). The CFD methods discretize Navier-Stokes equations on numerical meshes and solve the discretized equations to compute the macroscopic statistical variables such as the pressure and flow rate. The particle methods are mesh-free methods and treat the fluid as a series of discrete particulates. The flow regularity is obtained by tracing the movement of each particulate and analyzing their overall characteristics. The lattice Boltzmann method (LBM) (Mohamad, 2011) is a relatively new mesoscopic particle method. In this method, the virtual particulates move and collide at the lattice nodes of a fixed regular grid. In addition, it is easy to code and intrinsically parallelizable. Thus, the LBM method is particularly suitable for simulations of fluid flow in a complex zone, particularly one that is always changing because of the transport and deformation of PPG (Sukop et al., 2008; Chen et al., 2014).

When the suspensions of deformable PPG flow in the porous media, the particles usually gather at the inlet of the throats and generate high-concentration zones. The contacts among the PPG particles or with the porous media cannot be ignored. The discrete element method (DEM) (Cundall, 1971; Cundall and Strack, 1979; Zhu et al., 2008) is one of the most representative methods that address the particle contact. It traces all particles in the flow field at the Lagrange coordinate system and calculates the collision dynamics of each particle based on the deterministic particle contact model. Thus, the DEM has overwhelming superiority in studies on particle contact mechanisms. Purkait et al. (2005) established the deposit and restart model for particles immersed in the water considering the contact force between one particle with another or with the wall surfaces. McNamara and Falcon (2008) considered the contact deformation in their simulation studies on particle flow. Kruggel-Emden et al. (2008) improve the multi-particle DEM, and this modified method can simulate the collision process of particle clusters. This paper uses the DEM with a soft-sphere model to simulate the contact process among the PPG particles or with the porous media.

The coupling of fluid flow and particle movement is the key to simulating the flow of deformable PPG suspensions. Researchers have proposed many coupling methods such as the link-bounce-back method (LBB) (Ladd, 1994), dry-particle method (DPC) (Aidun et al., 1998) and immersed moving boundary method (IMB) (Noble and Torczynski, 1998). The LBB method is limited by the density ratio of solid to fluid, and its force and momentum computation is discontinuous. The DPC method involves removing or regenerating fluid nodes, which significantly increases the computational cost. The IMB method modifies the LBM with an additional collision operator, which depends on the percentage of the cell covered by solid particles. It was reasonably 
formulated from the mass and momentum balance perspective. Strack et al. (2007) indicates that IMB can obtain accurate and stable force and torque for solids moving through a lattice, even for small particle sizes relative to the computational grid size. Han and Cundall (2011) compare the sensitivity of LBB and IMB to the lattice resolution in a two-dimensional LBGK model. Results show that IMB is more accurate and less demanding on lattice resolution than LBB. These studies encourage me to choose the IMB to couple the LBM and DEM.

The coupling framework of LBM-DEM-IMB has been widely used to simulate the fluid-solid system (Cook, 2001; Strack et al., 2007; Ohtsuki and Matsuoka, 2010; Han and Cundall, 2011, 2013; Xiong et al., 2014; Zhang et al., 2014; Zhang et al., 2016). These papers mainly focus on rigid round particles. So the fluid-solid boundary and particle contacts are solved based on round or sphere assumptions. Since the particles are nondeformable, only one resultant force and the corresponding torque are needed for each particle. So the original implementation methods cannot deal with deformable particles.

As for the simulation of deformation dynamics, the boundary-integral algorithm and immersed boundary method (IB) are widely used. In the boundary-integral algorithm, the drop and solid surfaces are discretized and yield a large matrix for the unknown velocity on each element on the interface of each drop. The equations can be solved and then used to update the position and shape of each drop. This method has been successfully used to simulate the drops squeezing through constrictions (Rother et al.,2002; Zinchenko and Davis, 2006, 2017; Janssen and Anderson, 2007; Davis and Zinchenko, 2009). However, the high boundary discretization resolution and full rank coefficient matrix increase the computational cost, which limits its application in large-scale complex porous media.

The IB method was proposed by Peskin (1972). It has been used together with the lattice Boltzmann method (LBM) to simulate biological flows (Ma et al., 2014; Wei et al., 2014; Gross, 2014; Dash et al., 2015; Sun and Bo, 2015). In the IB-LBM system, the effect of moving boundary is realized by spreading the stress exerted by the boundary on the fluid onto the collocated grid points near the boundary. The velocity of the moving boundary nodes are interpolated from the adjacent fluid nodes with the Dirac function. The IB method based on the continuous forcing approach is widely used to address elastic boundary problems. However, this continuous force is difficult to determine and cannot be directly used in simulations of deformable PPG because of the large difference in density inside and outside the PPG.

Based on the above analysis, the paper introduces the discrete idea of the IB method into the coupling framework of LBM-DEM-IMB and develops a new implementation method which extends the application of 
the pre-existing DEM-LBM-IMB algorithm to deformable particle-fluid system. The improved method is firstly

119 validated by comparisons between simulation results and microscopic visualization experiments. Then, the 120 method is used to study the critical pressure gradient, where a PPG particle can deform and pass through a 121 relatively smaller throat.

\section{Numerical Method}

\subsection{Treatment method of deformation}

The IB method with the continuous forcing approach has been widely used to address elastic-boundary problems such as the flow of red blood cells. In this method, the red blood cell is treated as an elastic membrane filled with a fluid. The internal and external fluids are discretized into Euler variables, whereas the elastic membranes are discretized into Lagrange variables. The IB method handles the effect of the moving membranes by spreading the Lagrangian stress exerted by the boundary on the fluid onto the collocated Euler grid points near the boundary. The flow rate of the discretized membrane nodes is determined by interpolating from the

130 flow rates of the fluid points near the membranes with Dirac delta function. In these studies, the continuous

131 forcing approach requires deterministic equations, and their formulations depend on specific problems. For the present study on the flow of deformable PPG suspension, it is unreasonable to treat the PPG as capsules with similar densities inside or outside the membrane. It is also difficult to obtain the necessary deterministic equations of the body force in the IB method. Thus, the IB method cannot be directly used to treat the PPG deformation. This paper uses the boundary discrete idea of the IB method but introduces the accurate calculation of the solid-fluid interaction force in the IMB method instead of the interpolation and spreading operation in the original IB procedure.

According to the boundary discrete idea of the IB method, the boundary of each PPG in the porous media

139 is discretized into a set of pseudo particles such as the red spheres in Fig. 1. When the suspensions of 140 deformable PPG flow in the porous media, the boundary pseudo particles mainly interact with the fluid, other

141 suspended PPG, porous media and other pseudo particles. These interactions are quantified by an accurate force 142 analysis, which includes the interaction force between the fluid and the pseudo particles $\left(F_{f}\right)$, contact force of 143 the pseudo particles with other PPG or porous media $\left(F_{c}\right)$, and restoring force because of deformation $\left(F_{d}\right)$. The 144 calculation methods of these forces are described in the next sections. 


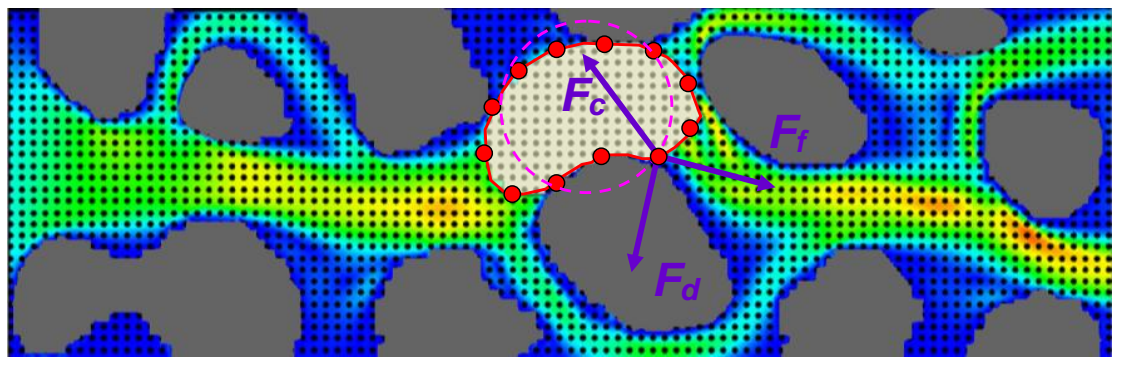

Fig. 1. Force analysis of deformed PPG in porous media.

(Gray area: rock matrix; Black points: LBM nodes; Yellow area: deformed PPG; Red spheres: discretized boundary pseudo particles; Pink circles: original shape of the PPG; Purple arrows: interaction forces)

After the interaction forces are obtained, the acceleration speed of each pseudo particle is calculated according to Newton's law:

$$
m a=\boldsymbol{F}_{f}+\boldsymbol{F}_{c}+\boldsymbol{F}_{d}
$$

where $m$ is the mass of each pseudo particle, which can be calculated by dividing the PPG mass by the number of pseudo particles; $\boldsymbol{a}$ is the acceleration speed of each pseudo particle.

After a period of $\Delta t$, the velocity and position of each pseudo particle are updated as

$$
\begin{aligned}
& \boldsymbol{v}=v_{\text {old }}+\boldsymbol{a} \Delta t \\
& \boldsymbol{x}=\boldsymbol{x}_{\text {old }}+\boldsymbol{v} \Delta t
\end{aligned}
$$

where $\boldsymbol{v}_{\text {old }}$ and $\boldsymbol{v}$ are the velocities before and after update, respectively; $\boldsymbol{x}_{\text {old }}$ and $\boldsymbol{x}$ are the positions before and after update, respectively.

When the interaction forces that act on each pseudo particle are different, the updated velocity and displacement of each boundary particle are different from one another. In other words, the PPG is deformed during the update of velocities and positions according to the accurate force analysis.

\subsection{Calculation of the interaction force $F_{f}$}

The interaction force between fluid and pseudo particles is simulated using the LBM with IMB modifications. The LBM is composed of evolution equations, an equilibrium distribution function and a discrete velocity model. The evolution equations reflect the time relaxation process of the density distribution function towards the equilibrium distribution function. There are two stages: collision and propagation, which are formulated as,

$$
f_{i}^{*}(\boldsymbol{x}, t)=f_{i}(\boldsymbol{x}, t)-\frac{\Delta t}{\tau_{B G K}}\left(f_{i}(\boldsymbol{x}, t)-f_{i}^{e q}(\boldsymbol{x}, t)\right)
$$




$$
f_{i}\left(\boldsymbol{x}+\boldsymbol{c}_{i} \Delta t, t+\Delta t\right)=f_{i}^{*}(\boldsymbol{x}, t)
$$
function,

$$
f_{i}^{e q}(\boldsymbol{x}, t)=\omega_{i} \rho\left[1+\frac{\boldsymbol{c}_{i} \cdot \boldsymbol{u}}{c_{s}^{2}}+\frac{\left(\boldsymbol{c}_{i} \cdot \boldsymbol{u}\right)^{2}}{2 c_{s}^{4}}-\frac{\boldsymbol{u}^{2}}{2 c_{s}^{2}}\right]
$$

where $\omega_{i}$ is the weighing factors; $\rho$ is the fluid density; $\boldsymbol{u}$ is the fluid velocity; $c_{s}$ is the lattice speed of sound.

The most widely used discrete velocity model is the DnQb model, where $n$ is the spatial dimension, and $b$ is the number of discrete velocity directions. The $D 2 Q 9$ model is used in the present paper. In this model, $c_{s}=c / \sqrt{3}$, and the nine possible velocity directions are

$$
\boldsymbol{c}=c\left[\begin{array}{ccccccccc}
0 & 1 & 0 & -1 & 0 & 1 & -1 & -1 & 1 \\
0 & 0 & 1 & 0 & -1 & 1 & 1 & -1 & -1
\end{array}\right]
$$

where $c=\Delta x / \Delta t ; \Delta x$ is the lattice spacing; $\Delta t$ is the time step.

The weighing factors of the $D 2 Q 9$ model are

$$
\omega_{i}=\left\{\begin{array}{cc}
4 / 9, & \boldsymbol{c}_{i}^{2}=0 \\
1 / 9, & \boldsymbol{c}_{i}^{2}=c^{2} \\
1 / 36, & \boldsymbol{c}_{i}^{2}=2 c^{2}
\end{array}\right.
$$

After the density distribution function is known, the macroscopic variables such as the fluid density, velocity and pressure are computed as follows,

$$
\begin{gathered}
\rho=\sum_{i=0}^{8} f_{i} \\
\rho \boldsymbol{u}=\sum_{i=0}^{8} \boldsymbol{c}_{i} f_{i} \\
P=c_{s}^{2} \rho
\end{gathered}
$$

In addition, the kinematic viscosity of the fluid satisfies the following equation,

$$
v=c_{s}^{2}\left(\tau_{B G K}-\frac{1}{2}\right) \Delta t
$$

The IMB method is used to address the solid-fluid interactions. In this method, the discrete nodes of the LBM should be marked as the fluid interior, fluid boundary, PPG interior and PPG boundary nodes, as shown in 
Fig. 2. The node type is determined by the number of intersection points between the ray line of a given node and the boundary segments of the PPG. If the number of intersection points is even, such as node A in Fig. 2, then the node is located outside the PPG. Otherwise, e.g., for node B in Fig. 2, the number of intersection points is odd; thus, it is located inside the PPG. The boundary nodes can be further determined starting from the initial one using the crawler algorithm (Ren et al., 2002), as shown in Fig. 2.

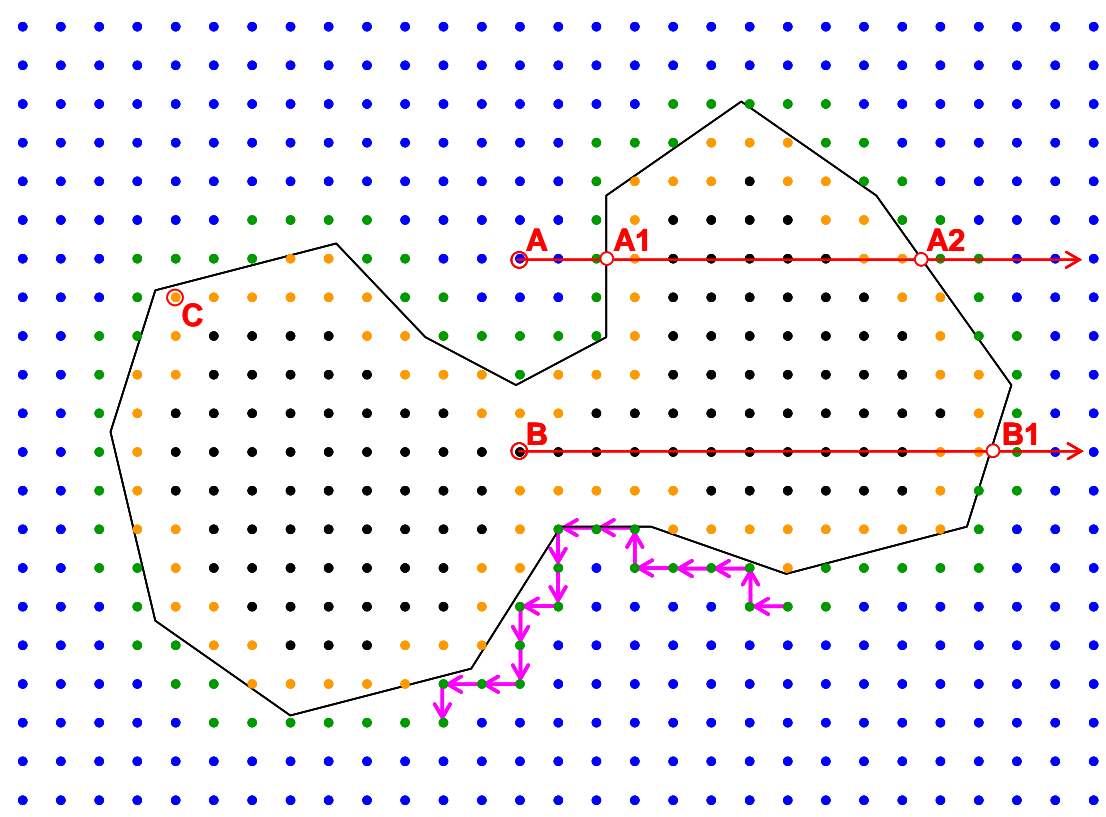

Fig. 2. Marking of LBM node types.

(Blue points: fluid interior; Green points: fluid boundary; Brown points: PPG boundary; Black points: PPG interior; Pink arrows: schematic of the crawler algorithm; Red arrows: the ray line from each node and the number of its intersections with the boundary segments of PPG are used to determine whether it is inside a PPG)

The IMB method introduces the solid volume fraction $\varepsilon_{s}$ to reflect the node types. For example, $\varepsilon_{s}$ of

node $\mathrm{C}$ in Figs. 2 and 3 is defined as the ratio of the gray subdivision volume covered by the PPG to that of the 


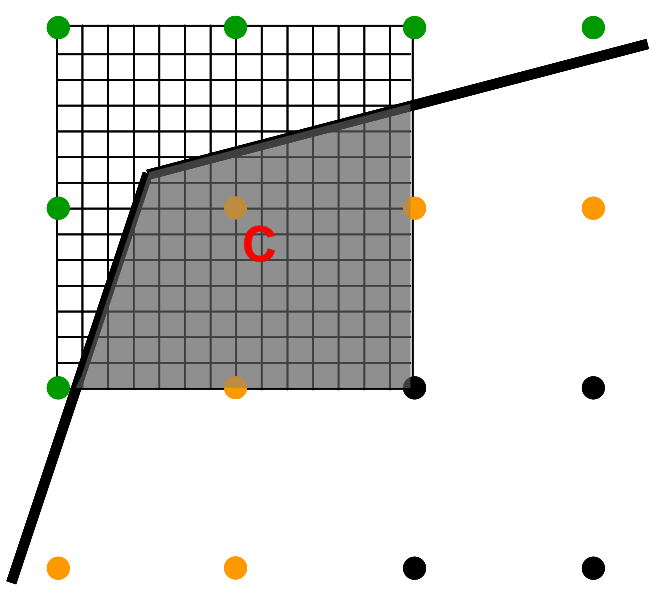

Fig. 3. Calculation of the solid volume fraction

(Blue points: fluid interior; Green points: fluid boundary; Brown points: PPG boundary; Black points: PPG interior; Black lines: real PPG boundary segments; Gray lattices: subdivision parts covered by PPG in the controlling cell surrounding node C; White

lattices: subdivision parts covered by the fluid)

After $\varepsilon_{s}$ of each node is determined, the solid-fluid interaction is accomplished by introducing an additional collision term into the evolution function of the original LBM. The equations are modified as,

$$
\begin{gathered}
f_{i}\left(\boldsymbol{x}+\boldsymbol{c}_{i} \Delta t, t+\Delta t\right)=f_{i}(\boldsymbol{x}, t)-\left(1-B_{s}\right)\left[\frac{\Delta t}{\tau_{B G K}}\left(f_{i}(\boldsymbol{x}, t)-f_{i}^{e q}(\boldsymbol{x}, t)\right)\right]+B_{s} \Omega_{i}^{s} \\
\Omega_{i}^{s}=\left[f_{-i}(\boldsymbol{x}, t)-f_{-i}^{e q}(\rho, \boldsymbol{u})\right]-\left[f_{i}(\boldsymbol{x}, t)-f_{i}^{e q}\left(\rho, \boldsymbol{v}_{p}\right)\right]
\end{gathered}
$$

where $-i$ denotes the opposite direction of $i ; \boldsymbol{u}$ and $\boldsymbol{v}_{p}$ are the velocities of the fluid and solid nodes at position $\boldsymbol{x}$, respectively.

$B_{s}$ is the weighting function of $\Omega_{i}^{s}$ and often calculated as,

$$
B_{s}\left(\varepsilon_{s}, \tau_{B G K}\right)=\frac{\varepsilon_{s}\left(\tau_{B G K} / \Delta t-1 / 2\right)}{\left(1-\varepsilon_{s}\right)+\left(\tau_{B G K} / \Delta t-1 / 2\right)}
$$

Based on the LBM method with IMB modifications, the interaction force between each pseudo particle and the fluid is calculated using the momentum theorem. It is written as,

$$
F_{f}=\frac{(\Delta x)^{2}}{\Delta t} \sum_{k=1}^{m} B_{s_{k}} \sum_{i=0}^{8} \Omega_{i}^{s} c_{i}
$$

where $m$ is the total number of PPG boundary nodes, which surround the segment from the midpoint between the pseudo particle and its left adjacent particle to the midpoint between the pseudo particle and its right adjacent particle.

\subsection{Calculation of contact force $F_{c}$}


211 using the DEM method based on the soft-sphere model. This method simplifies the contact actions to a 212 spring-damper process. Thus, the contact force is calculated by,

$$
\boldsymbol{F}_{c}=\boldsymbol{F}_{n} \boldsymbol{n}+\boldsymbol{F}_{t} \boldsymbol{t}
$$

213 where $\boldsymbol{n}$ and $\boldsymbol{t}$ are the normal and tangent unit vectors; $\boldsymbol{F}_{n}$ and $\boldsymbol{F}_{t}$ are the normal and tangent contact forces, 214 respectively. They are written as,

$$
\begin{aligned}
& \boldsymbol{F}_{n}=k_{n} \delta_{n}+c_{n} \frac{d \delta_{n}}{d t} \\
& \boldsymbol{F}_{t}=k_{t} \delta_{t}+c_{t} \frac{d \delta_{t}}{d t}
\end{aligned}
$$

where $k_{n}$ and $k_{t}$ are the normal and tangent contact stiffness, $c_{n}$ and $c_{t}$ are the normal and tangent damping factors, and $\delta_{n}$ and $\delta_{t}$ are the normal overlap and tangent displacement as shown in Fig. 4.

217 Special attention should be paid to the calculation of the overlap between one particle and the porous 218 media. Since the particle is much smaller compared with the porous media, their overlapping domain can be 219 treated as an arch. So we can firstly calculate the area of the overlapping domain and next calculate the height 220 of the arch iteratively. Then it can be used as the overlap to calculate the contact force between the particle and 221 the porous media.

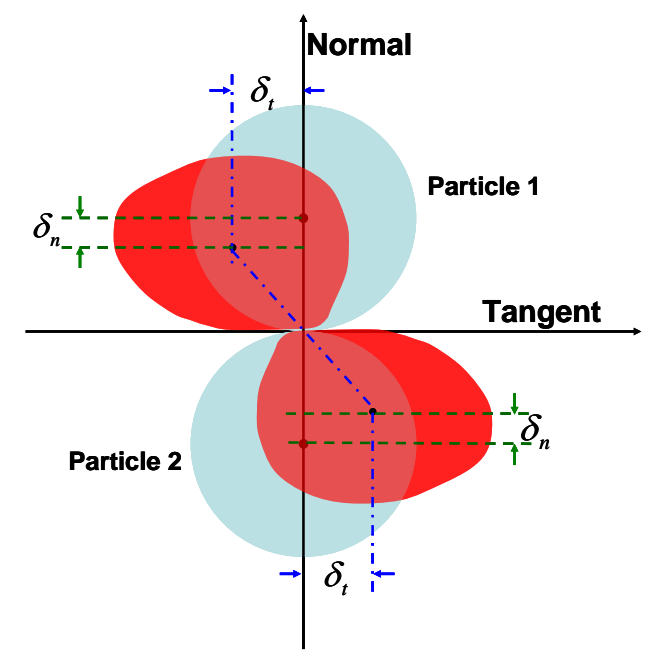

Fig. 4. Schematic of the particle contact.

\subsection{Calculation of restoring force $F_{d}$}

Since the PPG is elastic, there are forces resulting from the deformation, which makes the particle return to its original shape. The restoring force is calculated by, 


$$
\boldsymbol{F}_{d}=\boldsymbol{F}_{b} \boldsymbol{n}+\boldsymbol{F}_{s} \boldsymbol{t}
$$

227 where $\boldsymbol{n}$ and $\boldsymbol{t}$ are the normal and tangent unit vectors; $\boldsymbol{F}_{b}$ and $\boldsymbol{F}_{s}$ are the bending force and strain force, which 228 are formulated in detail as,

$$
\begin{aligned}
& \boldsymbol{F}_{\boldsymbol{b}}=E_{b}\left(\beta-\beta^{0}\right) \\
& \boldsymbol{F}_{s}=E_{s}\left(l_{a b}-l_{a b}^{0}\right)
\end{aligned}
$$

In Fig. 5, $\beta$ and $\beta^{0}$ are the vector angles of three adjacent pseudo particles before and after the

230 deformation; $l_{a b}$ and $l_{a b}^{0}$ are the distances between two adjacent particles before and after the deformation; $E_{b}$ and $E_{s}$ are the bending stiffness and strain stiffness, respectively.
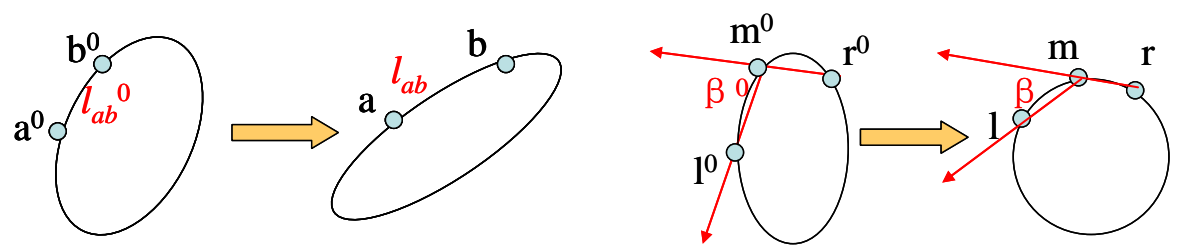

Fig. 5. Schematic of the particle deformation.

\section{Validation and Application}

\subsection{Experiment validation}

To validate the improved simulation method, the paper compares the simulation results with microscopic visualization experiments. In the experiments, the transparent micromodel was constructed from clear acrylic sheets, which include the lower plate, seal washer and upper cover plate (Yao et al., 2014). The diameters of the packed quartz sands are $149-841 \mu \mathrm{m}$, and their density is approximately $2.65 \mathrm{~g} / \mathrm{cm}^{3}$. The micron-size polyacrylamide elastic microspheres in the experiments are $16.49-63.62 \mu \mathrm{m}$, and their average diameter is approximately $27.44 \mu \mathrm{m}$. The density is approximately $1.01 \mathrm{~g} / \mathrm{cm}^{3}$. The deformable particle suspension is injected into the micromodel with a constant flow rate of $0.5 \mathrm{~cm}^{3} / \mathrm{min}$ and a concentration of $1000 \mathrm{mg} / \mathrm{L}$. The seepage flow of the deformable particle suspension was monitored and recorded using a KH-7700 microscope made by HIROX of America.

The deformable particles may be captured at the inlets of the throats with a smaller diameter than the particle size when the pressure difference across the throat is not sufficiently large. However, the typical characteristic of deformable particles is that they can deform and pass through the smaller throats when the pressure difference increases to a certain value. Thus, Fig. 6 shows typical images of the deformation process of 
an elastic particle. The size of the images is approximately $1100 \mu \mathrm{m} \times 800 \mu \mathrm{m}$. The smallest diameter of the throat is approximately $30 \mu \mathrm{m}$. For clear comparisons, we performed LBM-DEM simulations using identical

251 porous media. The deformable particle is injected from the upper inlet, and the fluid flow rate is approximately $0.02 \mathrm{~cm} / \mathrm{s}$. The initial shape of the particle is set as a sphere with a diameter of $40 \mu \mathrm{m}$. The elastic modulus of the particle is $58 \mathrm{~Pa}$. Fig. 7 shows the simulation results on the migration of the deformable particle through the 254 narrow throat. The pressure difference between lines $\alpha$ and $\beta$ in Fig. 7a was calculated.
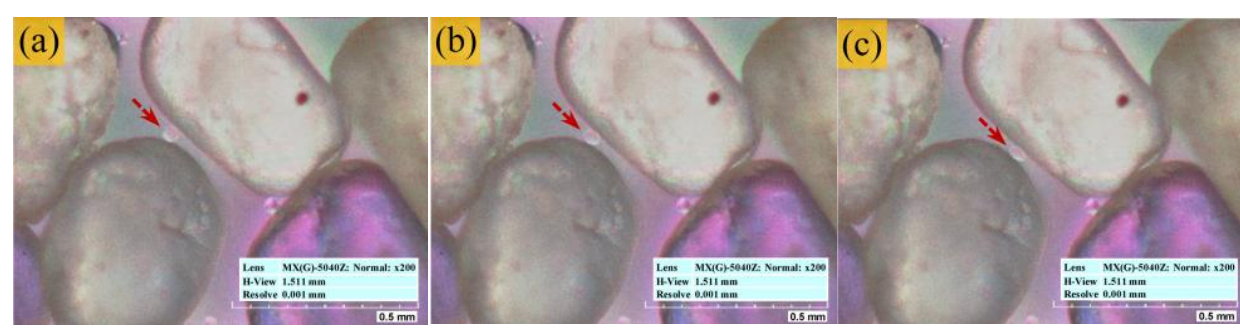

(d)

Fig. 6. Experiment results of deformable particle migration.

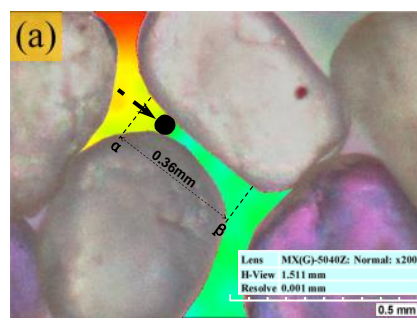

(b)

(c)
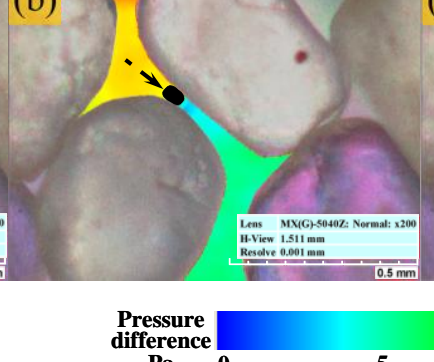

$\underset{P a}{0} \mathbf{0}$

Fig. 7. Simulation results of the deformable particle migration.

As shown in Figs. 6 and 7, the simulation results reproduce the migration and deformation process of the particle in the throat that the microscopic visualization experiments recorded. First, capture plugging occurs at the inlet of the throat because its diameter is smaller than the particle size. The pressure difference across the throat begins to increase at this stage as shown in Fig. 7a. When the pressure difference continues increasing, the particle begins to deform and enters the throat (Fig. 7b). Further down the narrow throat, the particle deforms to an ellipsoid and smoothly moves in the throat. During this stage, the pressure difference across the throat is maximized (Fig. 7c). Finally, after the particle moves out of the throat, it returns to its initial shape and size because of the restoring force. Simultaneously, the pressure difference between both ends of the throat rapidly decreases (Fig. 7d). Fig. 8 further quantifies the variation in pressure difference between lines $\alpha$ and $\beta$ when the deformable particle passes through the narrow throat. These analyses indicate that the simulation method reproduces the experimental phenomena and has the quantitative analysis advantage in critical conditions, where a particle can deform and pass through a narrow throat. 


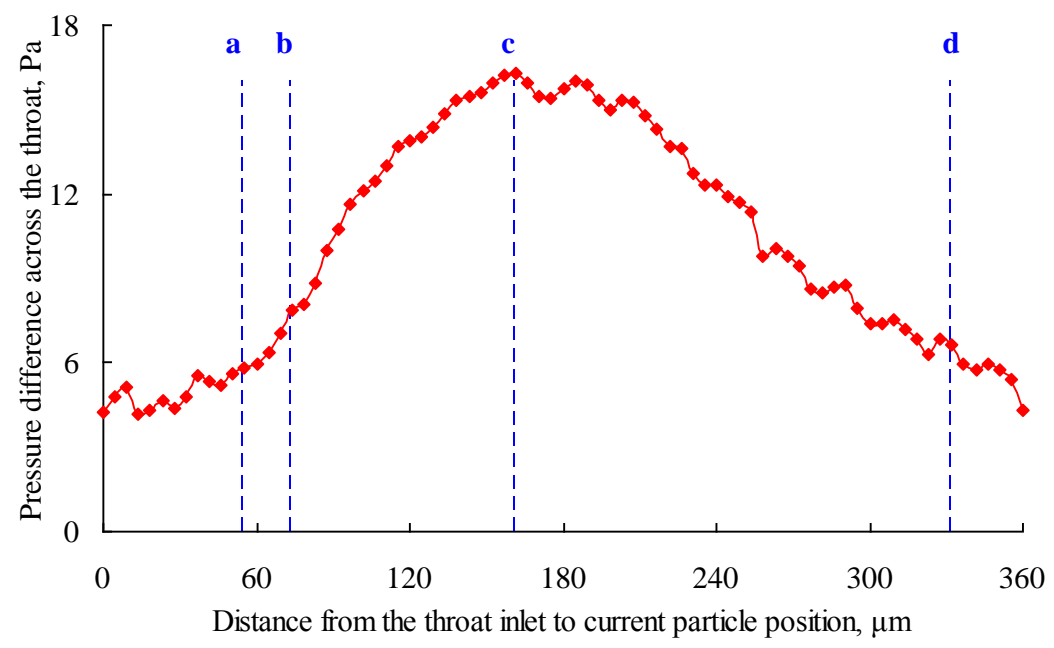

Fig. 8. Variation in pressure difference versus particle position in the throat.

\subsection{Application study}

As mentioned in Section 3.1, the pressure difference across the throat determines whether a particle can deform and pass through the throat or it is captured. Based on the improved method, we attempt to study the effect of the particle-throat diameter ratio, elastic modulus and fluid flow rate on the critical pressure gradient. Fig. 9 shows the simulation model. Its length is $700 \mu \mathrm{m}$. The largest and smallest diameters are $200 \mu \mathrm{m}$ and 100 $\mu \mathrm{m}$, respectively. The length of the narrowest throat part is $100 \mu \mathrm{m}$. There are $100-\mu \mathrm{m}$ transition regions at both sides of the throat. The upper and lower sides of the model are closed boundaries. The water is injected from the left inlet at a constant flow rate of $0.05 \mathrm{~cm} / \mathrm{s}$. In the study, only one PPG was simulated. Its original shape was set as a sphere with a diameter of $120 \mu \mathrm{m}$. The modulus of the PPG is $12 \mathrm{~Pa}$. The average pressure at the points $50 \mu \mathrm{m}$ from the inlet was recorded. The average pressure at other points $650 \mu \mathrm{m}$ from the inlet was also recorded. The pressure difference between two line groups was calculated.

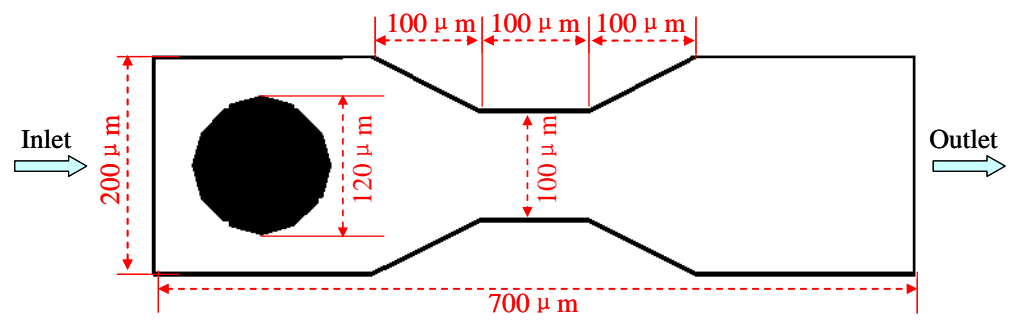

Fig. 9. Schematic of the simulation model.

Fig. 10 shows the variation in pressure difference when the PPG migrated in the pore-throat. As observed, the pressure difference remains relatively low before the PPG reaches the throat. However, it rapidly increases when the PPG moves into the throat and maximizes at approximately 4.5 Pa when the PPG reaches the middle 
part of the narrow throat. Then, the pressure difference gradually decreases when the PPG exits the throat.

288 Because of the vortex around the PPG at the throat outlet, there is a negative pressure difference, but it will 289 return to the normal value when the fluid flow becomes stable again. The difference between the highest and the 290 stable values is recognized as the plugging pressure difference, which is the smallest required value for a given 291 PPG to pass through a smaller throat. For example, in Fig. 10, the plugging pressure difference is approximately $2924 \mathrm{~Pa}$. More generally, the critical pressure gradient is used in simulation studies. It is calculated by dividing the 293 plugging pressure difference with the distance between the pressure-monitoring positions. Here, the critical 294 pressure gradient is approximately $4 \mathrm{~Pa} / 600 \mu \mathrm{m} \approx 0.007 \mathrm{MPa} / \mathrm{m}$.

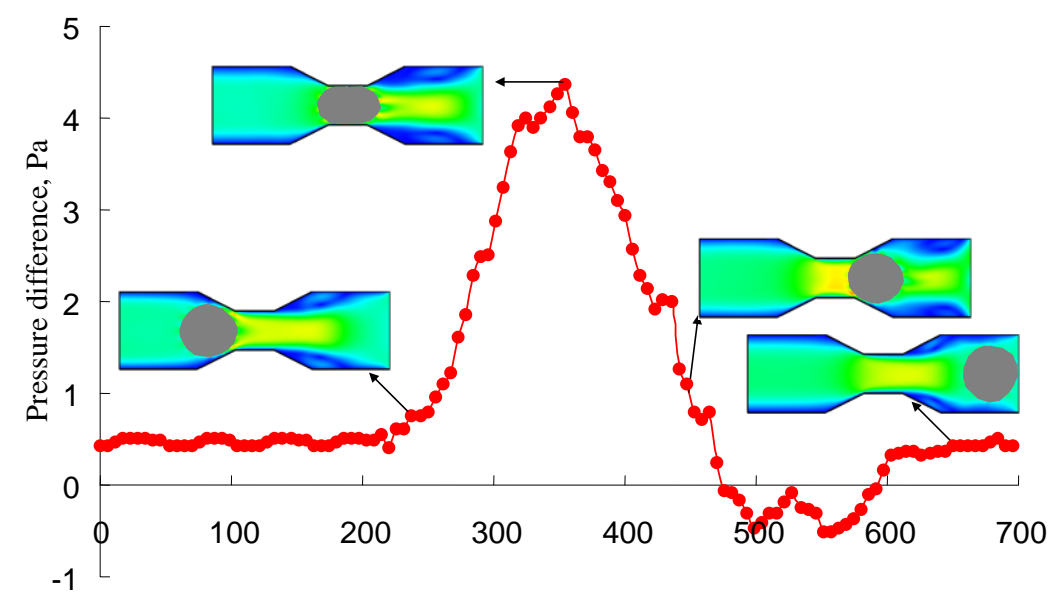

Distance from PPG to the left inlet, $\mu \mathrm{m}$

Fig. 10. Variation in pressure difference versus PPG position in the simulation model.

Figs. 11-13 show the effect of the particle-throat diameter ratio, elastic modulus and fluid flow rate on the critical pressure gradient. As observed, the critical pressure gradient rapidly increases with the particle-throat diameter ratio with a good exponential relationship. The critical pressure gradient also linearly increases with the elastic modulus of the PPG. However, as shown in Fig. 13, the effect of the fluid flow rate on the critical pressure gradient is negligible. These studies indicate that the critical pressure gradient is related to the properties of the PPG and pore-throat but not the fluid properties. 


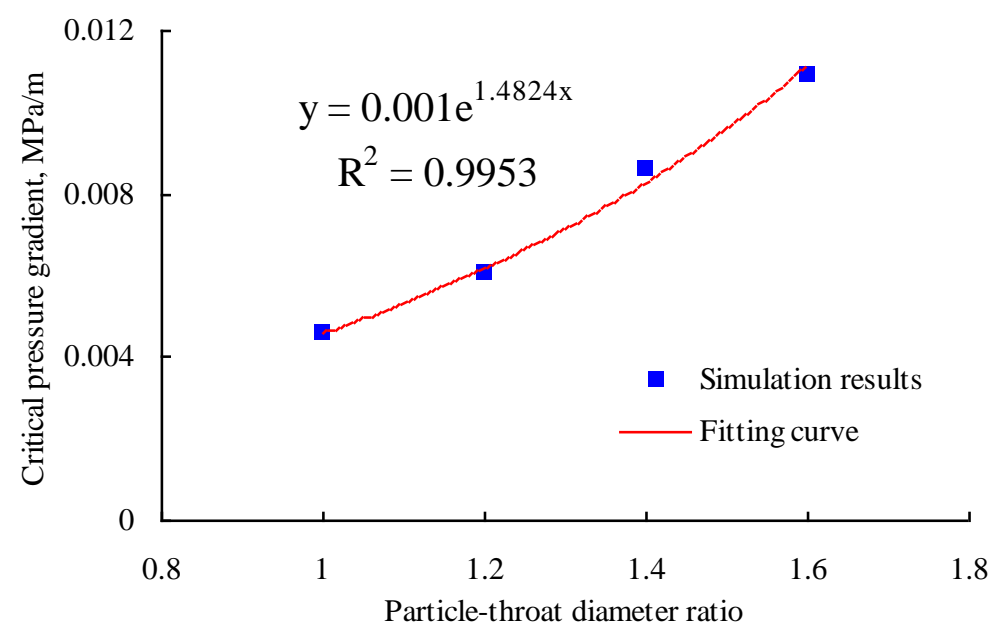

303

304

Fig. 11. Effect of the particle-throat diameter ratio on the critical pressure gradient.

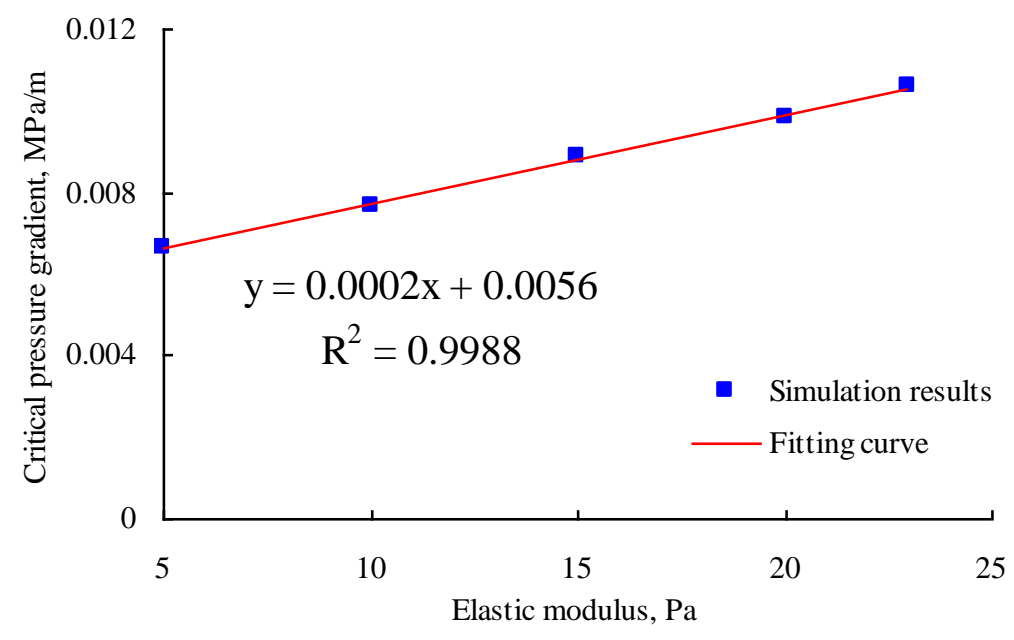

Fig. 12. Effect of the elastic modulus on the critical pressure gradient.

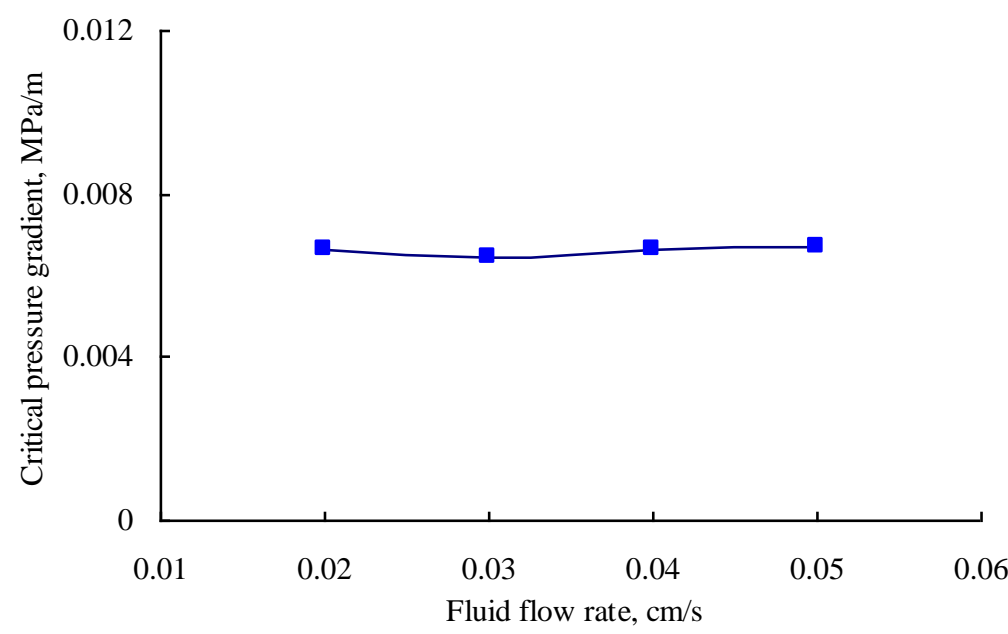

Fig. 13. Effect of the fluid flow rate on the critical pressure gradient.

To quantify the combined effect of the particle-throat diameter ratio and elastic modulus on the critical pressure gradient, 20 simulations were performed using different combinations of the two influence factors. On this basis, a quantitative mathematical model was established, as described in equation (20). Fig. 14 compares 
312 the simulated critical pressure gradients with the prediction results calculated by the established model. The

313 satisfactory fitting results validate the effectiveness and accuracy of the mathematical model. Thus, it can be

314 used as an important parameter in macroscopic simulations of PPG flooding for large field-scale problems.

$$
\nabla P=\left(28 E_{m}+0.00075\right) e^{\left(1.4 R_{r}\right)}
$$

315 where $\nabla P$ is the critical pressure gradient, $R_{r}$ is the particle-throat diameter ratio, and $E_{m}$ is the elastic 316 modulus of the PPG.

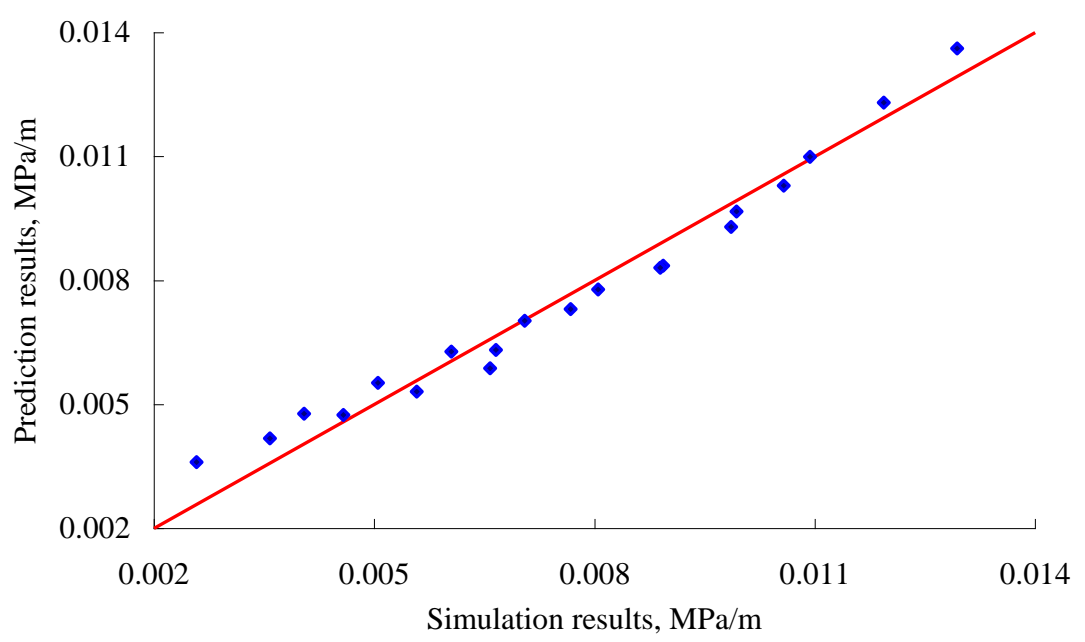

Fig. 14. Comparison of the critical pressure gradients between simulation and prediction results.

\section{Conclusions}

(1) An efficient LBM-DEM simulation method for the flow of the suspensions of deformable PPG in porous media has been improved.. The method combines the discrete idea of IB for particle deformation, LBM for fluid flow, DEM for particle contact and IMB for solid-fluid interaction. The transport, plugging and deformation of PPG in porous media are consistent between numerical simulations and microscopic visualization experiments, which validates the effectiveness of the improved. method. The improved. method can be used in many other engineering problems that involve the flow of suspensions of deformable particles in porous media. In addition, the deformable PPG is approximated by the polygon composed of the line segments between boundary discretization particles, so higher approximation precision needs increased discretization resolution and more computational cost. This limitation may affect the application of the improved method in large-scale problems.

(2) The critical pressure gradient has an exponential relationship with the particle-throat diameter ratio and a good linear relationship with the elastic modulus of the PPG. However, the fluid flow rate barely affects the 
critical pressure gradient. A multivariate mathematical model is proposed to quantify the relationships between the critical pressure gradient and the influence factors. Comparisons between simulation and prediction results prove the reliability of the mathematical model; thus, it can be used as the most important parameter in macroscopic simulations of PPG flooding for large field-scale projects.

\section{Acknowledgments}

The authors greatly appreciate the financial support of the National Natural Science Foundation of China (Grant No. 51574269), the Project supported by the National Science Foundation for Distinguished Young Scholars of China (Grant No. 51625403), the Important National Science and Technology Specific Projects of China (Grant No. 2016ZX05011-003), the Fundamental Research Funds for the Central Universities (Grant No. 15CX08004A), the National Natural Science Foundation of China (Grant No. 51604291) and the Natural Science Foundation of Shandong Province (Grant No. ZR2016EEB05).

\section{References}

Aidun, C.K., Lu, Y., Ding, E.J., 1998. Direct analysis of particulate suspensions with inertia using the discrete Boltzmann equation. J. Fluid Mech. 373, 287-311.

Bedrikovetsky, P., 2008. Upscaling of stochastic micro model for suspension transport in porous media. Transport Porous Med. 75 (3), 335-369.

Chen, S.G., Sun, Q.C., Jin, F., Liu, J.G., 2014. Simulations of Bingham plastic flows with the multiple-relaxation-time lattice Boltzmann model. Sci. China Phys Mech. 57 (3), 532-540.

Cook, B.K., 2001. A numerical framework for the direct simulation of solid-fluid systems. PhD thesis, Massachusetts Institute of Technology.

Cui, X., 2011. A study on the heterogeneous combination flooding system. Acta Petrolei Sinica 32 (1), 122-126.

Cundall, P.A., 1971. A computer model for simulating progressive large scale movements in blocky rock systems. Proceedings of the Symposium of the International Society of Rock Mechanics, Nancy, France, Vol. 1, Paper No. II-8.

Cundall, P.A., Strack, O.D.L., 1979. A discrete numerical model for granular assemblies. Geotechnique, 29 (1), 47-65.

Dash, S.M., Lee, T.S., Huang, H., 2015. Particle Sedimentation in a Constricted Passage Using a Flexible Forcing IB-LBM Scheme. Int. J. Comp. Meth. 12 (1), 1350095.

Davis, R.H., Zinchenko, A.Z., 2009. Motion of deformable drops through granular media and other confined geometries. J. Colloid Interf. Sci. 334 (2), 113-123.

Goudarzi, A., Zhang, H., Varavei, A., Taksaudom, P., Hu, Y., Delshad, M., Bai, B., Sepehrnoori, K., 2015. A laboratory and simulation study of preformed particle gels for water conformance control. Fuel 140, 502-513. 
Gross, M., Krüger, T., Varnik, F., 2014. Fluctuations and diffusion in sheared athermal suspensions of deformable particles. EPL $108(6), 68006$

Han, Y., Cundall, P.A., 2011. Resolution sensitivity of momentum - exchange and immersed boundary methods for solid-fluid interaction in the lattice Boltzmann method. Int. J. Numer. Meth. Fl. 67 (3), 314-327.

Han, Y., Cundall, P.A., 2013. LBM-DEM modeling of fluid-solid interaction in porous media. Int. J. Numer. Anal. Met. 37 (10), 1391-1407.

Imqam, A., Bai, B., 2015. Optimize the strength and size of preformed particle gels for better conformance control treatment. Fuel 148 (15), 178-185.

Janssen, P.J.A., Anderson, P.D., 2007. Boundary-integral method for drop deformation between parallel plates. Phys. Fluids 19 (4), 043602.

Kamal, M.S., Sultan, A.S., Almubaiyedh, U.A., Hussein, I.A., 2015. Review on Polymer Flooding: Rheology, Adsorption, Stability, and Field Applications of Various Polymer Systems. Polym. Rev. 21 (3), 1-40.

Kruggel-Emden, H., Rickelt, S., Wirtz, S., Scherer, V., 2008. A study on the validity of the multi-sphere Discrete Element Method. Powder Technol. 188 (2), 153-165.

Ladd, A.J., 1994. Numerical simulations of particulate suspensions via a discretized Boltzmann equation. Part 1. Theoretical Foundation. J. Fluid Mech. 271 (1), 285-309.

Liu, M.B., Liu, G.R., 2010. Smoothed Particle Hydrodynamics (SPH): an Overview and Recent Developments. Arch Comput Method Eng. 17 (1), 25-76.

Liu, Y., Hou, J., Wang, Q., Liu, J., Guo, L.L., Yuan, F., Zhou, K., 2017. Flow of pre-formed particle gel through porous media: A numerical simulation study based on the size exclusion theory. Ind. Eng. Chem. Res. 56 (10), 2840-2850.

Ma, J., Xu, Y., Tian, F., Tang, X. 2014. IB-LBM study on cell sorting by pinched flow fractionation. Bio-med. Mater. Eng. 24 (6), 2547-2554.

Mcnamara, S., Falcon, E., 2008. Simulations of dense granular gases without gravity with impact-velocity-dependent restitution coefficient. Powder Technol. 182 (2), 232-240.

Mohamad, A.A., 2011. Lattice Boltzmann Method- Fundamentals and Engineering Applications with Computer Codes. Springer, London.

Noble, D.R., Torczynski, J.R., 1998. A lattice-Boltzmann method for partially saturated computational cells. Int. J. Mod. Phys. C 9 (8), 1189-1201.

Ohtsuki, S., Matsuoka, T., 2010. A Study on the Effect of Particle Transport on Permeability in Porous Media by Using Hybrid LBM-DEM Simulation. Shigen-to-Sozai 126 (8/9), 503-511.

Purkait, M.K., Bhattacharya, P.K., De, S., 2005. Membrane filtration of leather plant effluent: flux decline mechanism. J. Membrane Sci. 258 (1-2), 85-96.

Raynal, L., Augier, F., Bazer-Bachi, F., Haroun, Y., Fonte, C.P.D., 2015. CFD Applied to Process Development in the Oil and Gas Industry - A Review. Oil Gas Sci. Technol. 29 (6), 949-954. 
Rother, M.A., Zinchenko, A.Z, Davis, R.H., 2002. A three-dimensional boundary-integral algorithm for thermocapillary motion of deformable drops. J. Colloid Interf. Sci. 245 (2), 356-364.

Ren, M., Yang, J., Sun, H., 2002. Tracing boundary contours in a binary image. Image Vision Comput 20 (2), 125-131.

Sang, Q., Li, Y., Yu, L., Li, Z., Dong, M., 2014. Enhanced oil recovery by branched-preformed particle gel injection in parallel-sandpack models. Fuel 136, 295-306.

Strack, O.E., Cook, B.K., 2007. Three-dimensional immersed boundary conditions for moving solids in the lattice-Boltzmann method. Int. J. Numer. Meth. Fl. 55 (2), 103-125.

Sukop, M.C., Huang, H., Lin, C.L., Deo, M.D., Oh, K., Miller, J.D., 2008. Distribution of Multiphase Fluids in Porous Media: Comparison between Lattice Boltzmann Modeling and Micro-X-Ray Tomography. Phys. Rev. E 77 (2), 026710.

Sun, D.K., Bo, Z., 2015. Numerical simulation of hydrodynamic focusing of particles in straight channel flows with the immersed boundary-lattice Boltzmann method. Int. J. Heat Mass Tran. 80, 139-149.

Wang, D., Dong, H., Lv, C., Fu, X., Nie, J., 2009. Review of Practical Experience by Polymer Flooding at Daqing. SPE Reserv. Eval. Eng. 12 (3), 470-476.

Wang, J., Liu, H., Wang, Z., Xu, J., Yuan, D., 2013. Numerical simulation of preformed particle gel flooding for enhancing oil recovery. J. Petrol. Sci. Eng. 112 (3), 248-257.

Wei, Q., Xu, Y.Q., Tian, F.B., Gao, T.X., Tang, X.Y., Zu, W.H., 2014. IB-LBM simulation on blood cell sorting with a micro-fence structure. Bio-med. Mater. Eng. 24 (1), 475-481.

Xiong, Q., Madadi-Kandjani, E., Lorenzini, G., 2014. A LBM-DEM solver for fast discrete particle simulation of particle-fluid flows. Continuum Mech. Therm. 26 (6), 907-917.

Yao, C., Lei, G., Cathles, L.M., Steenhuis, T.S., 2014. Pore-scale investigation of micron-size polyacrylamide elastic microspheres (MPEMs) transport and retention in saturated porous media. Environ. Sci. Technol. 48 (9), 5329-5335.

Yao, C., Lei, G., Hou, J., Xu, X., Wang, D., Steenhuis, T.S., 2015. Enhanced Oil Recovery Using Micron-Size Polyacrylamide Elastic Microspheres: Underlying Mechanisms and Displacement Experiments. Ind. Eng. Chem. Res. 54 (43), 10925-10934.

You, Z., Badalyan, A., Bedrikovetsky, P., 2013. Size-exclusion colloidal transport in porous media-stochastic modeling and experimental study. SPE J. 18 (4), 620-633.

Zhang, C., Jin, F., Chen, S., Xu, X., 2016. Flow behaviors of self-compacting concrete in rockfill gaps. J. Hydroelectric Eng. 35 (10), 112-120.

Zhang, H., Tan, Y., Shu, S., Niu, X., Trias, F.X., Yang, D., Li, H., Sheng, Y., 2014. Numerical investigation on the role of discrete element method in combined LBM-IBM-DEM modeling. Comput. Fluids 94 (2), 37-48.

Zhu, H.P., Zhou, Z.Y., Yang, R.Y., Yu, A.B., 2008. Discrete particle simulation of particulate systems: a review of major applications and findings. Chem. Eng. Sci. 63 (23), 5728-5770.

Zinchenko, A.Z., Davis, R.H., 2006. A boundary-integral study of a drop squeezing through interparticle constrictions. J. Fluid Mech. 564, 227-266.

Zinchenko, A.Z., Davis, R.H., 2017. Motion of Deformable Drops Through Porous Media. Annu. Rev. Fluid Mech. 49, 71-90. 